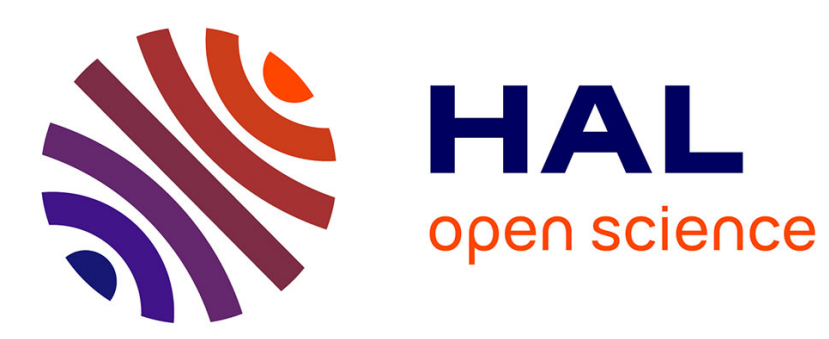

\title{
On the theory of phase transitions in organic superconductors
}

S. Brazovskii, V. Yakovenko

\section{To cite this version:}

S. Brazovskii, V. Yakovenko. On the theory of phase transitions in organic superconductors. Journal de Physique Lettres, 1985, 46 (3), pp.111-116. 10.1051/jphyslet:01985004603011100 . jpa-00232485

\section{HAL Id: jpa-00232485 https://hal.science/jpa-00232485}

Submitted on 1 Jan 1985

HAL is a multi-disciplinary open access archive for the deposit and dissemination of scientific research documents, whether they are published or not. The documents may come from teaching and research institutions in France or abroad, or from public or private research centers.
L'archive ouverte pluridisciplinaire HAL, est destinée au dépôt et à la diffusion de documents scientifiques de niveau recherche, publiés ou non, émanant des établissements d'enseignement et de recherche français ou étrangers, des laboratoires publics ou privés. 
Classification

Physics Abstracts

74.40

\title{
On the theory of phase transitions in organic superconductors
}

\author{
S. Brazovskii and V. Yakovenko
}

L. D. Landau Institute for Theoretical Physics, 117334 Moscow B334, Kosygina 2, U.S.S.R.

(Reçu le 2 juillet 1984, accepté le 17 décembre 1984)

\begin{abstract}
Résumé. - Les susceptibilités divergentes en dimension un, du type SDW, SS ou TS, donnent lieu à des phénomènes observables seulement si la cohérence de paires subsiste en présence d'effet tunnel inter-chaînes. Dans le régime sans gap, la cohérence peut être préservée par les déphasages électroniques entre les différentes chaînes qui peuvent être engendrés par des changements de symétrie. Celles-ci sont dues à des potentiels aléatoires ou oscillants pour un supraconducteur (SS ou TS) et à des champs magnétiques élevés pour SDW. Cette théorie nous permet de suggérer un modèle général du diagramme de phases des sels de Bechgaard où les divers états sont déterminés principalement par les changements de la symétrie cristalline.
\end{abstract}

\begin{abstract}
The 1d divergent susceptibilities such as SDW, SS or TS give rise to observable phenomena only if the pair coherence persists in the course of the interchain tunnelling. In the gapless regime it may be maintained by the proper interchain electronic phase shifts which can appear due to some symmetry changes. There are alternating or random potentials for superconductivity (SS or TS) and high magnetic fields for SDW. This theory permits us to suggest a general model for the phase diagram of the Bechgaard salts in a way that the variation of states is mainly determined by the crystal symmetry changes.
\end{abstract}

\section{Empirical picture.}

Organic compounds of the Bechgaard salts type (see [1] for a review) have a complicated phase diagram (PD) which includes the following states : metal (M), insulator (I), Peierls insulator (CDW), magnetic insulator (MI), antiferromagnetic (AF) insulator (SDW), a peculiar AF state in high magnetic field (H-SDW) and the superconducting (SC) singlet (SS) or, not excluded yet, triplet (TS) state. The variation of phases follows the changes of an anion type $\mathrm{X}$, anion structure (AS), pressure $P$, a magnetic field $H$ and a temperature $T$ (see the latests proceedings [2]).

In the present work we suggest a simple general model where the details of the PD are uniquely determined by the AS changes. The significance of the AS influence on the conducting stacks has been already underlined earlier $[5,6]$.

The experimental data [1-4] show us the following empirical correlation between the AS, characterized by the wave vector $\mathbf{Q}$, its influence on the conducting subsystem and the PD.

1) $\mathbf{Q}_{1}=(0,0,0)$. The intermolecule bonds are non-equivalent (dimerized), $P D$ : $\mathrm{M} \rightarrow \mathrm{MI} \rightarrow \mathrm{SDW}$. The last two phases almost coincide (F.E. $T_{\mathrm{MI}} \approx 20 \mathrm{~K}, T_{\mathrm{SDW}} \approx 10 \mathrm{~K}$ ). 
2) $\mathbf{Q}_{2}=\left(0, \frac{1}{2}, \frac{1}{2}\right)$. The molecules are non-equivalent (dimerized). PD : $\mathbf{M} \rightarrow \mathrm{MI} \rightarrow \mathrm{SDW}$ or CDW. $T_{\mathrm{MI}}$ and $T_{\mathrm{SDW}}$ are well separated. (F.E. in (TMTSF) ${ }_{2} \mathrm{SCN} T_{\mathrm{MI}}=160 \mathrm{~K}, T_{\mathrm{SDW}}=7 \mathrm{~K}$ ).

3) $\mathbf{Q}_{3}=\left(0, \frac{1}{2}, 0\right)-$ the neighbouring (contacting in b-direction !) stacks are non-equivalent. PD : M $\rightarrow$ SC $\rightarrow$ H-SDW. (The appearance under pressure of the $\mathbf{Q}_{3}$-type AS for the compounds that have a SC state only at $P \neq 0$ has already been suggested [3], while it has not yet been proved).

4) $\mathbf{Q}_{4}=\left(\frac{1}{2}, \frac{1}{2}, \frac{1}{2}\right)$. The stacks are tetramerized. PD : $\mathbf{M} \rightarrow I$. The transitions to $M I$ and $I$ states, unlike others, are driven externaly by the lowering of the crystal symmetry. Their temperatures coincide with that of the AS changes (cases 2 and 4) or they are determined as the crossover regions for the conductivity gap onset (case 1).

The rare case 2 helps us to fix the model for the whole family : a strongly correlated ld state with the charge and spin degrees of freedom separation in agreement with the bosonization theory [8]. The typical case 1 qualitatively corresponds to the same model, while the effect is very weak and may be interpreted controversially. The most interesting case 3 is considered in details below. The trivial case 4 is explained in any model.

\section{Theory.}

The PD of a 3d system of weakly interacting chains is usually supposed to correspond to the conditional 1d PD being defined by the divergences of the corresponding susceptibilities $\chi_{1 \mathrm{~d}}^{(i)}$ at $T \rightarrow 0$

$$
\chi_{1 \mathrm{~d}}^{(i)} \sim T^{-\beta_{i}}+\text { const. }, \quad \beta_{i}=2-\eta_{i}, \quad i=\mathrm{CDW}, \mathrm{SDW}, \mathrm{SS}, \mathrm{TS} .
$$

Given an appropriate interchain coupling $\lambda_{i}$ the total susceptibility would acquire the form :

$$
\begin{aligned}
& \chi^{(i)}=\chi_{1 \mathrm{~d}}^{(i)}+\lambda_{i}\left(\chi_{1 \mathrm{~d}}^{(i)}\right)^{2}+\cdots \approx\left[\left(\chi_{1 \mathrm{~d}}^{(i)}\right)^{-1}-\lambda_{i}\right]^{-1} \\
& \chi^{(i)}\left(T_{\mathrm{c}}\right)=\infty, \quad \chi_{1 \mathrm{~d}}^{(i)}\left(T_{\mathrm{c}}\right) \sim \lambda_{i}^{-1}, \quad \beta_{i}>0 .
\end{aligned}
$$

The problem is that the parameters $\lambda_{i}$ do not appear explicitly (except for the case $i=\mathrm{CDW}$ ) due to the loss of electronic coherence in the course of an interchain tunnelling. The effects of the symmetry lowering for cases 1 to 3 , by a magnetic field, and for some random potentials are just to maintain the coherence of Cooper or magnon pairs which restore the relations (2) for SDW or SC.

The only approach reflecting adequately all the basic features of $1 \mathrm{~d}$ theories is the bosonization method [8] generalized phenomenologically according to [9]. Following the convenient notations of [10] we shall define the phases $\theta_{n \pm}$ and $\varphi_{n \pm}$ according to the local gauge transformation of asymptotical $\pm k_{\mathrm{F}}^{(n)}$ counterparts $\psi_{\alpha \sigma}$ of electronic $\psi$-operator on the chain $n$ at the point $x$ for the spin projections $\sigma= \pm 1=\uparrow, \downarrow$ :

$$
\psi_{\sigma}(n, x)=\sum_{\alpha= \pm 1} \psi_{\alpha \sigma}(n, x) \exp \left\{i \alpha k_{\mathrm{F}}^{(n)} x+\frac{i}{2}\left(\alpha \theta_{n+}+\theta_{n-}+\sigma \alpha \varphi_{n+}+\sigma \varphi_{n^{-}}\right)\right\} .
$$

Given the system with high $T$ properties of a normal metal we use the zero order Hamiltonian $\mathrm{He}_{0}$

$\mathscr{H}_{0}=\sum_{\mathbf{k}}\left\{v_{\rho}(\mathbf{k})\left[\gamma_{\rho}(\mathbf{k})\left|\theta_{-}(\mathbf{k})\right|^{2}+\gamma_{\rho}^{-1}(\mathbf{k})\left|\theta_{+}(\mathbf{k})\right|^{2}\right]+v_{\mathrm{s}}\left[\gamma_{\mathrm{s}}\left|\varphi_{-}(\mathbf{k})\right|^{2}+\gamma_{\mathrm{s}}^{-1}\left|\varphi_{+}(\mathbf{k})\right|^{2}\right]\right\}$.

Here $\mathbf{k}=\left(k_{\|}, \mathbf{k}_{\perp}\right), \gamma_{\mathrm{s}} \sim 1$ is a constant since there are no long range interactions in the $\sigma$-channel, $\gamma_{\rho}=\gamma_{\rho}(\mathbf{k})$ takes into account all $1 \mathrm{~d}$ and long range $3 \mathrm{~d}$ interactions [13]. We neglect here, for 
the sake of briefness, the frequency dispersions (they are described naturally in the Lagrangian form of (4)) approximating $\gamma_{\rho}(\omega, \mathbf{k}) \approx \gamma_{\rho}\left(\mathbf{k}_{\perp}\right)$ with different values of $\gamma_{\rho}\left(\mathbf{k}_{\perp}\right)$ in separated regions $\omega \gtrless \omega_{\text {ph. }}$. The dispersion $\gamma_{\rho}$ is physically inherent for the systems with the SC since it takes into account an interchain e-e attraction at some $(\omega, \mathbf{k})$ regions were $\gamma_{\rho}(\omega, \mathbf{k})<1$. In the following we shall also admit, for the sake of briefness, that $v_{\rho}=v_{\mathrm{s}}=v_{\mathrm{F}}^{*}=$ const. We shall take the units where $\hbar=1, v_{\mathrm{F}}^{*}=1, a=1$ ( $a=$ intermolecular spacing on this stack).

Other $3 \mathrm{~d}$ interactions as well as the effects of various symmetry lowerings are reflected by the Hamiltonian $\mathcal{H}_{1}$ (see Refs. [5, 6, 8, 10, 13])

$$
\begin{aligned}
\mathscr{H}_{1}=\int \mathrm{d} x \sum_{n}\left\{\begin{array}{r}
g_{3} \\
\cos \left(2 \tilde{\theta}_{n+}(x)+v\right)
\end{array}\right. \\
\quad+t_{\perp} \sum_{\alpha, \sigma}\left[\psi_{\alpha \sigma}^{+}(n+1, x) \psi_{\alpha \sigma}(n, x) \times\right. \\
\left.\exp \left\{i\left[\left(g+(-1)^{n} \alpha \kappa\right) x+\mu_{n}(x)-\mu_{n+1}(x)\right]\right\}+\text { c.c. }\right]+ \\
\left.\quad+\sum_{m} g_{m n} \cos \left(\tilde{\theta}_{n+}-\tilde{\theta}_{m+}\right) \cos \varphi_{n+} \cos \varphi_{m+}\right\} \\
\tilde{\theta}_{n+}(x)=\theta_{n+}(x)+(-1)^{n} \kappa x+\mu_{n}(x) ; \quad \partial \mu_{n} / \partial x=\gamma_{\rho} v_{\rho}^{-1} V_{n}(x) .
\end{aligned}
$$

Here $g_{3}$ and $v$ are the amplitude and the phase of the Umklapp scattering (for the cases 1 and 2 $g_{3}=g_{3}^{(1,2)}$ and $v=v^{(1,2)}$ respectively and $\left.g_{3}^{(2)} \gg g_{3}^{(1)}, v^{(2)}-v^{(1)}=\pi / 2\right), g_{m n}$ is the interchain backward scattering amplitude, $t_{\perp}$ is the interchain tunnelling matrix element, $V_{n}(x)$ is a random potential for a system with disorder, $\kappa=\left|k_{\mathrm{F}}^{(n+1)}-k_{\mathrm{F}}^{(n)}\right|, \kappa \neq 0$ for the case $3, g=(e / c) b H$, $b=|\mathbf{b}|$ is an interstack spacing, such that $\mathbf{b} \perp \mathbf{H}$, where $\mathbf{H}$ is a magnetic field, $|\mathbf{H}|=H$. The parameters $\mu_{n}, \kappa, \dot{g}$ in (5) appear naturally as a conventional gauge transformation of (3) under external fields.

The conditions for the phases $\mathbf{M}$ and $\mathrm{MI}$ to exist lead to inequalities $\gamma_{\mathrm{s}} \geqslant 1, \bar{\gamma}_{\rho}<1$, $\left(\bar{f}=\left\langle f\left(\mathbf{k}_{\perp}\right)\right\rangle\right)$.

We shall need the correlation functions, being calculated with the Hamiltonian $\mathscr{H}_{0}$ :

$$
\begin{aligned}
& G=\left\langle\psi_{n \alpha}(0) \psi_{n \alpha}^{+}(z)\right\rangle \sim|z|^{-\eta_{F}}, \quad z=x+i t, \quad \eta_{F}=2-\beta_{\mathrm{F}} \\
& K_{n_{1} n_{2} n_{3} n_{4}}^{\sigma \sigma^{\prime}}\left(z_{1}, z_{2}, z_{3}, z_{4}\right)=\left\langle\psi_{\sigma+}\left(z_{1}, n_{1}\right) \psi_{\sigma+}^{+}\left(z_{3}, n_{3}\right) \psi_{\sigma^{\prime}-}\left(z_{2}, n_{2}\right) \psi_{\sigma^{\prime}}^{+}\left(z_{4}, n_{4}\right)\right\rangle \\
& K^{\sigma \sigma^{\prime}}=K_{n m m}^{\sigma \sigma^{\prime}}\left(z_{1}, z_{2}, z_{3}, z_{4}\right) \sim\left[\left|z_{1}-z_{3}\right|\left|z_{2}-z_{4}\right|\right]^{-\eta_{F}}\left[\frac{\left|z_{1}-z_{2}\right|\left|z_{4}-z_{3}\right|}{\left|z_{1}-z_{4}\right|\left|z_{3}-z_{2}\right|}\right]^{\delta} \\
& \eta_{\mathrm{F}}=\frac{1}{4}\left(\bar{\gamma}_{\rho}+\bar{\gamma}_{\rho}^{-1}+\gamma_{\mathrm{s}}+\gamma_{\mathrm{s}}^{-1}\right) ; \quad \delta=\delta\left(\mathrm{s}, \mathrm{s}^{\prime}\right)=\frac{1}{4}\left[\bar{\gamma}_{\rho}^{-1}-\bar{\gamma}_{\rho}+\sigma \sigma^{\prime}\left(\gamma_{\mathrm{s}}^{-1}-\gamma_{\mathrm{s}}\right)\right] .
\end{aligned}
$$

We have omitted in equations (6) and (7) the $z$-phase dependent factors.

The expressions (1) for $\chi_{1 \mathrm{~d}}^{(i)}$ follow from (7) if we tie up the ends $z_{j}$ by pairs and integrate over them. But it was just the artificial tying up of the ends which happens at the MF approach that usually brought generally mistaken results (2). To clarify this question we shall consider here the expansion of susceptibilities in terms of the Hamiltonian (5). Let us consider for example the first order term in series of $t_{\perp}^{2}$ for any $\chi^{(i)}$, shown in figure 1. Following this diagram with the help of equations (4), (5) and (7) we find easily the unique expression $\left({ }^{1}\right)$

$\chi^{(i)}(T)=\frac{1}{T^{2-\eta_{i}}}+t_{\perp}^{2} \int_{|z|,|u|,|v|<T^{-1}} \frac{\mathrm{d}^{2} z \mathrm{~d}^{2} u \mathrm{~d}^{2} v f_{i}(u, v)}{|u-v|^{2 \eta_{\mathrm{F}}-\eta_{i}}[|u||v||z-u||z-v|]^{\eta_{i} / 2}}+\cdots$

( $\left.{ }^{1}\right)$ We can derive (8) even without application to (5) appealing only most general scaling hypothesis governed by the five independent indexes $\left(\eta_{i}, \eta_{F}\right)$ instead of two $\left(\delta, \eta_{F}\right)$ in (7). 


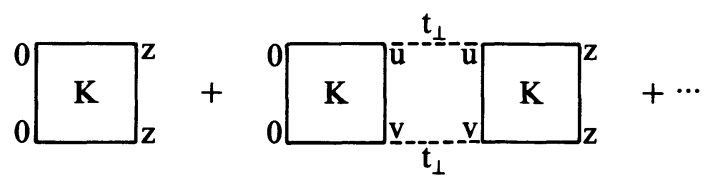

Fig. 1. - The expansion of a correlation function in series of $t_{\perp}$.

The function $f_{i}=f_{i}(u, v)$ in (8) depends on the character of the symmetry lowering otherwise $f=$ const. We can distinguish the two cases A and B.

A) The integration over $(u-v)$ in (8) is divergent being limited only by the temperature cutoff $T^{-1}$. Then the second term in (8) at any $i$ is of the order of $T^{-\beta_{i}} \varepsilon$, where

$$
\varepsilon \sim\left(t_{\perp} / T^{\beta}\right)^{2} \sim\left(T_{3 \mathrm{~d}} / T\right)^{\beta_{\mathrm{F}}}, \quad T_{3 \mathrm{~d}} \sim t_{\perp}^{1 / \beta_{\mathrm{F}}}, \quad \beta_{i}=2-\eta_{i},
$$

and the series are expanded in powers of the non-specific (i.e., independent on $i$ ) parameter (9). In this general case the hypotheses of equation (2) is not correct and the $1 \mathrm{~d}$ PD is not reproduced. Moreover the 1d PD seems to have no relation to any 3d physical quantity. At $T \sim T_{3 \mathrm{~d}}, \varepsilon \sim 1$, there is a crossover to the $3 \mathrm{~d}$ regime studied in [11], where the band theory conclusions can be qualitatively applicable. We shall only note that there are no small parameters in this region so that $T_{\mathrm{c}}$ for SDW, SS, TS cannot be smaller than $T_{3 \mathrm{~d}}$.

B) The integrals over $(u-v)$ in (8) are convergent at some length $\xi$ such that $|u-v| \lesssim|\xi|<T_{3 \mathrm{~d}}^{-1}$. In this case the intermediate ends $u, v$, which correspond to the tunnelling points, are tied up at the scale $\xi$. Then additionally to the series in $\varepsilon$ (see Eq. (9)) we find a subseries of the type (2) with the effective coupling constant $\lambda_{i}$ :

$$
\lambda_{i} \sim t_{\perp}^{2} / \xi^{\beta_{i}-2 \beta_{\mathrm{F}}}, \quad T_{\mathrm{c}} \sim \xi^{-1}\left(t_{\perp} \xi^{\beta_{\mathrm{F}}}\right)^{2 / \beta_{i}} .
$$

Before the symmetry lowering effects the convergence in (8) can only be provided by the inequality $2 \eta_{F}-\eta_{i}-2>0$, i.e. $\beta_{i}-2 \beta_{F}>0$, when we find $\xi \sim 1$ (atomic scale of the pair) and the condition $T_{\mathrm{c}} \gg T_{3 \mathrm{~d}}$ is fulfilled automatically. Such a situation, in principle, is not excluded for strong $3 \mathrm{~d}$ long range repulsions. (Note that for free fermions $\beta_{i}-2 \beta_{\mathrm{F}}=-2$ ). We shall not discuss here this case as well as the extremely $1 \mathrm{~d}$ case $\beta_{\mathrm{F}}<0$ when $T_{3 \mathrm{~d}}=0$. In these cases the CDW or SDW instabilities would dominate independently on the symmetry (we cannot anticipate an equivalently strong attraction when a SC would dominate). The picture as a whole will be discussed elsewhere. In the following $\beta_{\mathrm{F}}>0,2 \beta_{\mathrm{F}}-\beta_{i}>0$.

Let us consider the symmetry of the cases 1,2 , when the gap $\Delta$ appears in the charge channel $[8,12]$. Then a function $f_{i}=f(w), w=u-v$, appears in (8) for $i=\mathrm{SDW}, \mathrm{CDW}$ :

$$
\begin{aligned}
f_{i} & =f_{i}(w) \sim|w \Delta|^{2 \eta_{F}-\eta_{i}} \mathrm{e}^{-2 \Delta|w|} \quad \text { at } \quad|w| \gg \Delta^{-1}, \\
w & =u-v \quad \Delta \sim g_{3}^{\left(2-\bar{\gamma}_{\rho}\right)^{-1}} \gg T_{3 \mathrm{~d}} .
\end{aligned}
$$

An analogous situation has already been considered for the system with an onchain attraction $\left(\gamma_{s}<1\right)$ which results in a gap in the spin channel [13]. We arrive at the results (10) with $\xi \sim \Delta^{-1}$. The formation of $\Delta$ shows up itself as a MI crossover transition with $T_{\mathrm{c}}$ as a subsequent one to the CDW or SDW phase. The cases 1 and 2 are distinguished only quantitatively : 2) $\Delta=\Delta_{2} \gg T_{3 \mathrm{~d}} \gg T_{\mathrm{c}}$; 1) $\Delta=\Delta_{1} \sim T_{3 \mathrm{~d}} \gtrsim T_{\mathrm{c}}$.

It is important to note that here and in the following, for all cases with $\beta_{i}-2 \beta_{\mathrm{F}}<0$, we find from (10) that $T_{\mathrm{c}}<T_{3 \mathrm{~d}}$, unlike the case with $\beta_{i}-2 \beta_{\mathrm{F}}>0$. But here are no contradictions since at $\Delta>T_{3 \mathrm{~d}}$ or generally at $\xi^{-1}>T_{3 \mathrm{~d}}$, the scale $T_{3 \mathrm{~d}}$ has no sense and the condition $\Delta>T_{\mathrm{c}}$ is sufficient to reach the regime (10). 
Let us consider case 3 in the limit when the chain's inequivalence is strong enough so that $\kappa \gg \Delta$. Now the onchain commensurability is lost, the gap $\Delta$ disappears and the MI phase is eliminated. The functions $f_{i}$ in various channels have the forms

$$
\begin{aligned}
& i=\mathrm{CDW}, \mathrm{SDW}: f_{i}=\cos (2 \kappa(x+y)) ; \quad i=\mathrm{SS}, \mathrm{TS}: f_{i}=\cos (2 \kappa(x-y)), \\
& x=\operatorname{Re} u, \quad y=\operatorname{Re} v ; \quad \kappa \gg \Delta ; \quad \kappa \gg T_{3 \mathrm{~d}} .
\end{aligned}
$$

Now there is no convergence over $(u-v)$ for $i=\mathrm{CDW}$, SDW. Then the SDW instability can no longer develop until down to the renormalized $3 \mathrm{~d}$ crossover temperature $T_{3 \mathrm{~d}}^{*}=T_{3 \mathrm{~d}}^{2} / \kappa$. The suppression from $T_{3 \mathrm{~d}}$ down to $T_{3 \mathrm{~d}}^{*}$ is due to the interchain incommensurability $\delta k_{\mathrm{F}}= \pm \kappa$.

On the contrary, for $i=\mathrm{SS}$, TS the oscillating factor (12) improves the convergence of the integral in (8). Now we only need that for $i=\mathrm{SS}$ or $i=\mathrm{TS}$ a rather general inequality $2 \eta_{\mathrm{F}}-\eta_{i}>0$ i.e. $2 \beta_{\mathrm{F}}-\beta_{i}<2$ is fulfilled which is only slightly more rigorous than the necessary condition $\beta_{i}>0$. We arrive at the results (10) with $\xi=\kappa^{-1}$. Due to the "attraction " between the tunnelling points $u$ and $v$, the scale $\xi=\kappa^{-1}$ plays the role of the Cooper pair length. This case would correspond to the quasi-1d SC and manifest the fluctuating $1 \mathrm{~d}$ regime. At the same time there will be no pseudogap in the spectrum resolving a number of contradictions (see [1]).

The random potentials $V_{n}(x)$ in (5) produce locally the same effect as $\kappa$. Introducing the interchain random correlation length $l$ according to $\left\langle V_{n}(x)\left[V_{n}(y)-V_{n+1}(y)\right]\right\rangle=l^{-1} \delta(x-y)$, we arrive at (8) for $i=\mathrm{SS}, i=\mathrm{TS}$ with $f_{i} \sim \exp (-|x-y| / l)$ and then at (10) with $\xi=l$. So the shallow random potentials stimulate the SC while they suppress the SDW or CDW.

The effect of SDW or CDW stabilization at high $H$ is explained analogously to SC at $\kappa \neq 0$. Without the symmetry lowerings 1 to 4, we find from equations (5) and (8) at high $H$ the result (12) with the change $y \rightarrow-y$ and changing from $2 \kappa$ to $q$. For $i=\mathrm{CDW}$, SDW we again arrive at (10) with $\xi=q^{-1}$, being interpreted as the scale of the magnon pair in the course of the tunnelling. For the materials under discussion the situation is complicated by the fact that the phase $\mathrm{H}$-SDW is observed only for the AS 3 i.e., at $\kappa \gg q$. Consequently, the H-SDW appears as a double SDW with the wave vector $Q_{n}=2 k_{\mathrm{F}}+2 \kappa(-1)^{n}$ alternating from chain to chain. It is reflected in a more complicated form of $f_{i}$ for $i=\mathrm{CDW}$, SDW : $f_{i}=\cos (2 \kappa(x+y)) \times$ $\cos (q(x-y))$. The $3 \mathrm{~d}$ coupling appears now only at the order of $t_{\perp}^{4}$, so that we have to consider diagrams of double the order than that of figure 1. Finally, we find the results of (1) and (10) with the constant of « indirect exchange » $\lambda_{i}^{*}, i=\mathrm{SDW}, \mathrm{DCW}$ :

$$
\begin{aligned}
\lambda_{i} & \rightarrow \lambda_{i}^{*}=\left.\lambda_{i}^{2} \chi_{1 \mathrm{~d}}^{(i)}\right|_{T \sim \kappa} ; & \lambda_{i}=t_{\perp}^{2} / q^{2 \beta_{\mathrm{F}}-\beta_{i}} ; & 2 \beta_{\mathrm{F}}-\beta_{i}>2 \\
T_{\mathrm{c}} & \sim\left(\lambda_{i}^{*}\right)^{1 / \beta_{i}} \sim \lambda_{i}^{2 / \beta_{i}} / \kappa ; & i & =\mathrm{SDW}, \mathrm{CDW} .
\end{aligned}
$$

\section{Conclusions.}

Quasi one-dimensional conductors with the SC phase transition, both organic and $\mathbf{M X}_{3}$, have a definite common property (the new family [15] has not yet been studied in this respect). This is the incommensurability combined (in contrast to TTF-TCNQ, etc.) to the single type of carriers. Theoretically, this peculiarity can be related to two phenomena : the pinning's breakdown and the stimulation of the Cooper pair coherence (closely related to the second phenomena is the restoration of the SDW at high magnetic fields). After the critical depinning value $\kappa_{0} \sim \Delta$ is exceeded by the incommensurability parameter $\kappa$, a finite $T_{\mathrm{c}} \neq 0$ for the SC appears, but then decreases at higher $\kappa$ (see Eq. (10) at $\xi=\kappa^{-1}, 2 \beta_{\mathrm{F}}-\beta_{i}>0$ ). This picture agrees with the observable dependence $T_{\mathrm{c}}(p)$ if we assume (following Ref. [3]) that the AS of case 3 appears and further develops under pressure. It is interesting to note here a plausible agreement with 
the phenomenological theory [16]. The interpretation of the interplay between so called Rand Q-states (see [1-4]) in terms of our theory is straightforward. The anion disorder in the Q-state affects the PD indirectly just suppressing the crystallographic transition to the AS of case 3 and preserving the structure of case 1 .

\section{References}

[1] Jérome, D., Schulz, H., Adv. Phys. 31 (1982) 239.

[2] "Conducteurs et supraconducteurs synthétiques à basse dimension ", J. Physique Colloq. 44 (1983) C3.

[3] Moret, R., Pouget, J. P., Comes, R., Bechgaard, K., ibid., p. 957.

[4] Pouget, J. P., Moret, R., Comes, R., Shirane, G., Bechgaard, K., Fabre, J. M., ibid., p. 969.

[5] BrazovskiI, S., Gordunin, S., Sov. Phys. JETP Lett. 31 (1980) 371.

Barišić, S., BrazovskiI, S., Recent developments in Condensed Matter Physics (Plenum, N.Y.) 1 (1981) 141.

[6] Emery, V. J., Bruinsma, R., Barišić, S., Phys. Rev. Lett. 48 (1982) 1039.

[7] TORRANCE, J. in [2], p. 799.

[8] Luther, A., Peschel, J., Phys. Rev. 139 (1974) 291.

Luther, A., EMery, V. J., Phys. Rev. Lett. 33 (1979) 764.

[9] Efetov, K. B., Larkin, A. J., Sov. Phys. JETP 42 (1975) 390.

[10] Suzumura, Y., Prog. Theor. Phys. 61 (1979) 1.

[11] Prigodin, V. N., Firsov, Y. A., Sov. Phys. JETP 49 (1979) 369.

[12] Dzjaloshinskii, I. E., Larkin, A. I., Sov. Phys. JETP 34 (1972) 422.

[13] Gutfreund, H., Klemm, R. A., Phys. Rev. B 14 (1976) 1073.

[14] GoR'Kov, L. P., LeBed', A. G., J. Physique Lett. 45 (1984) L-433.

[15] Yagubskit, E. B., Schegolev, I. F., Laukhin, B. H., Konopovich, P. A., Kirtzovnik, M. V., ZvaRykina, A. V., Buravov, L. I., Zh. Eksp. Teor. Fiz. Pis'ma 39 (1984) 12.

[16] Efetov, K. B., Sov. Phys. JETP 54 (1981) 583, 1198. 